\title{
EFFICACY OF TAILORED RECOMMENDATIONS TO PROMOTE HEALTHY LIFESTYLES: A POST-HOC ANALYSIS OF A RANDOMIZED CONTROLLED TRIAL
}

María Barroso ${ }^{1,2,3}$, M. Dolors Zomeño ${ }^{4,5}$, Jorge L. Díaz ${ }^{1}$, Silvia Pérez ${ }^{6,7}$, Ruth MartíLluch $^{8}$, Ferran Cordón ${ }^{9}$, Rafel Ramos ${ }^{8,10,11}$, Carmen Cabezas ${ }^{12}$, Gemma Salvador ${ }^{12}$, Conxa Castell ${ }^{12}$, Helmut Schröder, ${ }^{4,13}$, María Grau ${ }^{1,13,14 *}$

${ }^{1}$ Cardiovascular Epidemiology and Genetics Research Group, IMIM-Hospital del Mar Research Institute, Barcelona, Spain

2 Department of Pediatrics, Obstetrics, Gynecology and Preventive Medicine, School of Medicine, Autonomous University of Barcelona, Spain

${ }^{3}$ Eastfield Health, Ashburton, New Zealand

${ }^{4}$ Cardiovascular Risk and Nutrition, IMIM-Hospital del Mar Medical Research Institute, Barcelona, Spain

${ }^{5}$ School of Health Sciences, Blanquerna-Ramon Llull University, Barcelona, Spain

${ }^{6}$ Regicor Research Group, IMIM-Hospital del Mar Medical Research Institute, Barcelona, Spain

${ }^{7}$ Consortium for Biomedical Research in Cardiovascular Disease (CIBERCV), Barcelona, Spain

${ }^{8}$ Unitat de Suport a la Recerca de Girona, Institut Universitari d'Investigació en Atenció Primària Jordi Gol, Girona, Spain

${ }^{9}$ Centre d'Atenció Primària Montilivi, Direcció d'Atenció Primària Girona, Institut Català de la Salut, Girona, Spain

${ }^{10}$ Department of Medical Sciences, School of Medicine, University of Girona, Spain

${ }^{11}$ Girona Biomedical Research Institute, Girona, Spain

${ }^{12}$ Catalan Agency of Public Health, Barcelona, Spain

${ }^{13}$ Consortium for Biomedical Research in Epidemiology and Public Health (CIBERESP), Barcelona, Spain

${ }^{14}$ Serra Húnter Fellow, Department of Medicine, University of Barcelona, Barcelona, Spain

Correspondence: María Grau; Department of Medicine; School of Medicine and Health Sciences, \#143 Casanova St. 08036 - Barcelona, Spain; Email mariagrau@ub.es

Acknowledgments: The authors wish to thank Marta Cabañero, Leny Franco, Neus Guday, Montse Peris, Martina Sidera, and Susanna Tello for their contribution to the data collection and management of this project and appreciate the revision of the English text by Elaine Lilly, $\mathrm{Ph} \mathrm{D}$. This study was financed by Spain's Ministry of Economy and Competitiveness through the Carlos III Health Institute FEDER (CM12/03287, CPII17/00012, and FIS14/00449).

Conflict of Interest: The authors declare that they have no conflict of interest. 


\section{Compliance with Ethical Standards}

Ethical approval: All procedures performed in studies involving human participants were in accordance with the ethical standards of the institutional and/or national research committee and with the 1964 Helsinki declaration and its later amendments or comparable ethical standards. The clinical trial protocol was registered at ClinicalTrials.gov (\#NCT02373319) and approved by the Institutional Research Board [Clinical Research Ethic Committee of the Parc de Salut Mar (CEIC-PSMAR, \#2014/5815/I)].

Informed Consent: Informed consent was obtained from all individual participants included in the study. 


\begin{abstract}
Prevention is the key to stopping the ravages of cardiovascular diseases, the main cause of death worldwide. The objective was to analyze the efficacy of tailored recommendations to promote healthy lifestyles. Secondary analysis of a parallel-arm randomized controlled trial with 1-year follow-up. Individuals aged 35-74 years from Girona (Spain) randomly selected from a population with no cardiovascular diseases at baseline were included. Participants in the intervention group received a brochure with tailored healthy choices according to the individual risk profile and a trained nurse explained all recommendations in detail in a 30-minute consultation. One-year changes in smoking, Mediterranean diet adherence, physical activity and weight were analyzed with McNemar, Student t, Wilcoxon and Fisher exact tests according to an intention-totreat strategy. Of 955 individuals [52.3\% women; mean age $50( \pm 10)$ years] randomly allocated to the intervention or control group, one participant in each group presented a cardiovascular event and 768 (81\%) were reexamined at 1-year follow-up. The prevalence of nonsmokers increased in both the intervention and control groups $(78.1 \%$ to $82.5 \%, \mathrm{p}=<0.001$, and 76.7 to $78.8 \%, \mathrm{p}=0.015$, respectively); however, significance persisted only in the intervention group when stratified by sex, age group, and educational level. Adherence to a Mediterranean diet increased in the intervention group $(22.3 \%$ to $26.5 \%, \mathrm{p}=0.048)$. In conclusion, a brief personalized intervention with science-based recommendations according to individual risk profiles appears to improve healthy lifestyles, particularly nonsmoking and adherence to a Mediterranean diet. This promising intervention system offers evidence-based recommendations to develop healthy lifestyles.
\end{abstract}


Keywords: Cardiovascular Diseases; Empowerment; Lifestyles; Mediterranean Diet; Physical Activity; Prevention 


\section{BACKGROUND}

Prevention is the key to stopping the ravages of cardiovascular diseases (CVD), the main cause of death worldwide [1] and characterized as diseases of long duration and generally slow progression. The adoption of a healthy cardiovascular lifestyle (i.e., healthy diet pattern, moderate alcohol consumption, nonsmoking, normal weight, and regular physical activity) could prolong life expectancy at the age of 50 years by 14 and 12 years for women and men, respectively [2].

The Health Belief Model, a psychosocial health behavior change model, suggests that individual beliefs about health problems, perceived benefits of action and barriers to action, self-efficacy, and the cue to action explain the health-promoting behavior [ 3 , 4]. For instance, the burden of potentially modifiable risk factors has been positively correlated with the individual's perceived need to improve his or her physical health [5, 6]. Thus, individual empowerment linked to health literacy is an important mechanism for self-management to maintain or improve health outcomes, while also lowering the primary health care professional's workload and generating cost-effective gains for health care delivery.

Several systematic reviews have assessed the role of computer-tailored solutions on physical activity, dietary behavior change, and smoking cessation [7, 8, 9]. We hypothesize that a self-screening method to estimate individual cardiovascular risk that provides tailored recommendations to promote healthy changes in user behaviors, could encourage users to take responsibility for their own health and well-being $[10,11]$. The aim of this study was to analyze the efficacy of such method to promote healthy choices in four areas: (1) increased adherence to the Mediterranean diet, (2) increased physical activity, (3) reduced weight, and (4) nonsmoking. The secondary objective was to 
analyze whether the self-screening system's efficacy differed by sex, age, and educational level.

\section{METHODS}

\section{Study Design and participants}

The study design, methodology, and population have been reported previously $[10,11]$. Briefly, individuals aged 35-74 years, residing in the city of Girona and surrounding area (northeastern Spain) and with no cardiovascular diseases at baseline were included in this randomized parallel-arm controlled trial with a 12-month follow-up. The participants in the intervention group in this received personalized preventive recommendations to promote healthy choices according to each individual's cardiovascular risk profile (blood pressure levels, lipid profile, anthropometry, diabetes, and smoking habit), dietary pattern, and physical activity level. The comparison group received the standard communication of results (i.e., report of all physiological and selfcollected variables). All participants were duly informed and provided written informed consent before enrollment. The clinical trial protocol was registered at ClinicalTrials.gov (\#NCT02373319) and approved by the Institutional Research Board [Clinical Research Ethic Committee of the Parc de Salut Mar (CEIC-PSMAR, \#2014/5815/I)].

\section{Measures}

At baseline, a team of trained nurses collected the following data using validated devices: height (OMRON® BF-214) and weight (to estimate body mass index), blood pressure (OMRON® $\left.{ }^{\mathrm{M} 3}\right)$, lipid profile (total, high-density and low-density cholesterol 
and triglycerides), glycaemia and glycated hemoglobin (ABXHoriba, Montpellier, France). In addition, self-administered standard questionnaires were used to gather data on sex, age, educational level, tobacco consumption, and self-reported hypertension, hypercholesterolemia, or diabetes and related treatment.

Adherence to the Mediterranean diet was measured with the 14-item Mediterranean Diet Adherence Score (MEDAS) questionnaire, validated for the Spanish population [12]. The questionnaire consists of 12 questions on food consumption frequency and 2 questions on food intake habits considered characteristic of the Spanish Mediterranean diet. Each question was scored 0 or 1; final score ranged from 0 to 14 [12].

The REGICOR questionnaire includes 6 two-part questions that collect information on the 4 dimensions of physical activity (type of activity, frequency, duration, and intensity). To estimate total energy expenditure in leisure time physical activity, the intensity assigned to each activity considered in the questionnaire was multiplied by the number of days it was performed in a month and by the average number of minutes per day. The metabolic equivalent of task (MET) for the 6 activities considered were as follows: walking (4), brisk walking (5), gardening (5), walking trails (6), climbing stairs (8), and any sport activity (10) [13].

Intervention: the tailored recommendations

The tailoring process started with the creation of an individual profile for each participant from the collected data: (1) prevalence of cardiovascular risk factors; (2) tobacco use (yes/no); and (3) physical activity performance level. 
The recommendations provided to the intervention group were based on the most current scientific evidence $[14,15,16]$. These participants received a packet with the results of baseline examinations (blood pressure, lipid profile, diabetes, smoking), and their estimated cardiovascular risk, using the Framingham-REGICOR risk function validated for the Spanish population [17]. A trained nurse explained the personalized recommendations in detail in a 30-minute consultation about approaches to improve cardiovascular health by controlling weight, quitting smoking, improving adherence to the Mediterranean diet, and increasing physical activity based on individual performance levels (sedentary, moderate, vigorous). The comparison group received only a standard letter reporting the results of baseline examinations (blood pressure, lipid profile, diabetes, smoking). The recommendations to improve adherence to Mediterranean diet and to control weight have been published elsewhere [11]. Appendix A includes the pyramids for physical activity in individuals aged $<65$ and $\geq 65$ years.

\section{Follow-up and outcomes}

All participants were reexamined by the same team of nurses at 1-year follow-up and all variables were collected again. Those who had experienced any cardiovascular event were excluded.

The outcomes considered were one-year changes toward healthier behaviors in the four dimensions of the study design: (1) Adherence to Mediterranean diet, defined as $<9$ points at baseline and $\geq 9$ at follow-up on the 14-item MEDAS questionnaire [18]; (2) Normal weight according to the World Health Organization criteria [19], using a body mass index category $<18.5$ or $\geq 25 \mathrm{~kg} / \mathrm{m}^{2}$ at baseline and $\geq 18.5$ and $<25 \mathrm{~kg} / \mathrm{m}^{2}$ at followup; (3) Smoking, assessed dichotomously as individuals who smoked at baseline and 
did not at follow-up; and (4) Physical activity, a change defined as individuals with a sedentary way of life at baseline (daily energy expenditure in moderate or vigorous physical activity $<750$ or $<420 \mathrm{kcal}$, respectively), who reported moderate or vigorous physical activity at follow-up [13].

\section{Data Analysis}

Normality of distribution was tested for all continuous variables, which were presented as mean and standard deviation or median and interquartile range when their distribution departed from normal. Categorical variables were presented as proportions.

Data were analyzed according to an intention-to-treat strategy. Missing values were imputed using baseline observation carried forward for participants who did not attend the reexamination, were known to be alive and did not present with any cardiovascular event in the follow-up. The percentage of individuals in each group were plotted with healthy behaviors at baseline and at 12-month follow-up. To estimate for both groups whether this percentage differed significantly before and after the intervention (within groups analysis), McNemar tests were used. The mean and the standard deviation for body mass index and MEDAS questionnaire results were also calculated. Student t-tests were applied to ascertain the differences in both variables in the intervention and comparison groups. In addition, median and interquartile ranges were used to describe the energy expenditure in physical activity; differences were assessed with Wilcoxon tests. To evaluate the efficacy of the system in different subgroups, the analysis was stratified by sex, age ( $<50$ and $\geq 50$ years) and by educational level as a proxy of socioeconomic status. Fisher exact tests were performed to estimate the differences between 
the intervention and comparison groups. A sensitivity analysis was performed following a per protocol strategy including only individuals with follow-up data available [20].

All statistical analysis was performed with the R Statistical Package (R Foundation for Statistical Computing, Vienna, Austria; V.3.5.1, July 2018).

\section{RESULTS}

This post hoc analysis included 955 individuals who were randomly assigned and 953 received the assigned treatment (475 and 478 in the intervention and comparison groups, respectively). At 1-year follow-up, 768 participants (383 and 385, respectively) were reexamined (overall response rate $=81 \%$ ). The primary analysis was designed to assess the effectiveness of tailored recommendations on improving total cholesterol. For the sample size in this study, differences $\geq 0.4( \pm 2)$ units in the MEDAS Score are considered statistically significant with an alpha and beta risk of 0.5 and 0.2 , respectively. One individual in each group presented with a CVD event during followup (Figure 1). There were no significant differences between the two randomized groups (Table 1).

The percentage of individuals reporting a Mediterranean diet pattern (MEDAS $\geq 9$ points) increased significantly from baseline to one-year follow-up in the intervention group $(22.3 \%$ to $26.5 \%)$. The change was nonsignificant in the comparison group $(20.1 \%$ to $21.5 \%)$. Both groups experienced a significant increase in the mean MEDAS score: $7.1( \pm 2.0)$ to $7.2( \pm 1.9)$ and $6.9( \pm 1.9)$ to $7.2( \pm 1.9)$ points in the intervention and comparison groups, respectively (Figure 2). The prevalence of nonsmokers was significantly increased in both groups, but the magnitude of the effect was higher in the intervention group and, when stratified by sex, age, and educational level, the change 
was significant in all subgroups of participants. In contrast, no comparison subgroup showed significant differences for this variable (Table 2 and 3). Finally, the median of the energy expenditure in physical activity significantly decreased for the individuals in the comparison group [1871 (interquartile range: $923-3163)$ to 1650 (747-3073) $\mathrm{kcal} /$ day], together with a significant decrease of individuals with daily energy expenditure in moderate $(\geq 750 \mathrm{kcal})$ or vigorous $(\geq 420 \mathrm{kcal})$ physical activity $(69.2 \%$ 64.4\%); no significant difference was observed in the intervention group [1935 (9743343 ) to 1846 [923 to 3490$) \mathrm{kcal} / \mathrm{day}$ ] and (67.4\%-64.2\%) (Figure 2). There were no differences between groups for any variable in the stratified or non-stratified analyses. The per-protocol sensitivity analysis, including only individuals with follow-up data available, showed similar results (Supplementary Table 1 and Supplementary Figure 1).

\section{DISCUSSION}

An intervention based on the Health Belief Model, with personalized recommendations designed to promote healthy habits according to the individual's cardiovascular risk profile, could be particularly effective to improve adherence to the Mediterranean diet and nonsmoking behavior. These personalized recommendations were automatically produced by a validated methodology for the self-screening of cardiovascular risk [10]. The system delivers science-based, tailored recommendations according to the individual profile.

\section{Tailoring to improve adherence to health promotion recommendations}

The findings suggested that a brief intervention with tailored dietary recommendations that taps into individual beliefs about health problems and self-management could 
contribute to a successful lifestyle change [3, 4]. Adherence to the Mediterranean diet, with its known benefits in preventing CVD [21], is an achievable target, based on the positive results observed just 12 months after a single brief intervention providing individually tailored dietary counseling. This could be a feasible and successful strategy to implement in healthy populations. However, the Mediterranean diet is not the pattern in many countries in the world because of the different agricultural and rural models. Indeed, diets link worldwide human health with environmental sustainability. Thus, providing an increasing world population with a healthy and sustainable diet (i.e. appropriate caloric intake, a diversity of plant-based foods, low amounts of animal source foods, unsaturated rather than saturated fats, and small amounts of refined grains, highly processed foods, and added sugars) represents a major challenge [22].

The finding that personalized smoking cessation counselling was successful is consistent with two systematic reviews showing that interactive and tailored Internetbased interventions can moderately increase smoking abstinence [23]. Most such interventions have been delivered through intensive short-term text-messaging, smoking cessation apps, computer-based interventions or even through Instant Messaging [24, 25]. Several authors have suggested that removing "superfluous information" by personalizing recommendations improves adherence, and have commented that communication strategies must consider the characteristics of each target group for which interventions are designed (e.g., teens vs. adults in the workforce or the older population) [iError! Marcador no definido., 24, 25]. In our study, the tailored message indicating the beneficial effect of smoking cessation had a significant effect in all subgroups analyzed. The sum of all recommendations given (e.g., increased adherence to the Mediterranean diet, increased physical activity, reduced weight, and 
nonsmoking) likely produced a holistic message that facilitated healthy choices, and smoking cessation in particular. Nevertheless, achieving high levels of user engagement remains a major challenge, as the objective is long-lasting smoking abstinence.

\section{Tailored information to improve the efficacy of preventive messages}

The multi-level complexity of a tailored computer-based intervention was summarized in the Consolidated Framework for Implementation Research that identified the factors influencing its implementation [26]. This system for the cardiovascular self-screening and recommendation of preventive activities presents three of the main characteristics recommended for an appropriate system: (1) low complexity that facilitates its use, (2) adaptability to fit the local context, and (3) compatibility with the National Health Systems because of the potential to be used as a monitoring system [27]. Basically, the use of tailoring of individual assessments and feedback aims to eliminate superfluous information and ensure that the remaining recommendations are personally relevant and useful to help the user stay motivated, increase personal empowerment and health literacy, and enact and sustain desirable lifestyle changes [28]. This system fits with the Health Beliefs Model that attempts to explain health behavior and health behavior change by focusing on the individual with the principal intention of providing information either to improve knowledge or change behavior [3, 4].

Despite the tailoring process, we found that the percentage of individuals with a nonsedentary way of life decreased in participants aged $\geq 50$ years, pointing out that our message needed to be more specific for this subgroup. In the intervention group, this prevalence also increased in those having completed only a secondary education; there was no apparent explanation for this finding. Indeed, increasing the complexity of 
interventions could make it difficult to ascertain whether any improvements observed were secondary to the tailoring component of the intervention per se [29]. Conway et al., in a systematic review, concluded the need for new studies in which the role of tailoring could be isolated from other interventions [30].

\section{Limitations}

The main goal of the present study was to motivate healthy people to engage in CVD prevention. However, the use of intermediate variables as study outcomes may be a limitation because of the low consistency, stability, and sensitivity to small changes, compared with hard endpoints. In addition, most of the outcomes considered are selfreported (adherence to Mediterranean diet, energy expenditure in physical activity, or smoking) and hence subject to respondent bias. Individuals in the intervention group differed significantly in adherence to the Mediterranean diet and nonsmoking behavior from baseline to 1-year follow-up; although this was not observed in the comparison group, the between-group difference was nonsignificant. The limited sample size is the most likely explanation of this lack of statistical significance. Hospital admissions and deaths were registered, but the number of events was low because of participant characteristics and length of follow-up (1 year). Beyond the effectiveness of the recommendations themselves, the Hawthorne effect could have had a role in the outcomes reported, as participants may change their behaviors because they feel observed. This is an intrinsic bias of all randomized controlled trials, but the impact is low because the effect can be similar in both intervention and comparison groups. Finally, the ethnic group of participants was not registered; however, ethnic minorities constitute an estimated $12 \%$ of the population in the reference area. Since this is a 
population-based sample and participants were randomized to intervention and comparison groups, this variable is not likely to act as a confounder. However, future studies should have enough statistical power to assess differences by ethnic group when this variable is available.

\section{CONCLUSIONS}

A brief personalized intervention with science-based recommendations according to individual risk profiles might improve healthy lifestyles, particularly adherence to a Mediterranean diet and nonsmoking. This promising system offers an innovative, personalized way of delivering scientifically proven tools to facilitate the development of healthy lifestyles. Further studies are needed to evaluate the long-term effects and cost-effectiveness of this intervention in the general population and also in individuals with chronic diseases, with sufficient power to assess the differences between intervention and comparison groups. 
Appendix A. Personalized recommendations on physical activity given to each participant in the intervention group according to the individual profile: (A) Physical activity recommendations for individuals aged $<65$ years and (B) Physical activity recommendations for individuals aged $\geq 65$ years

A
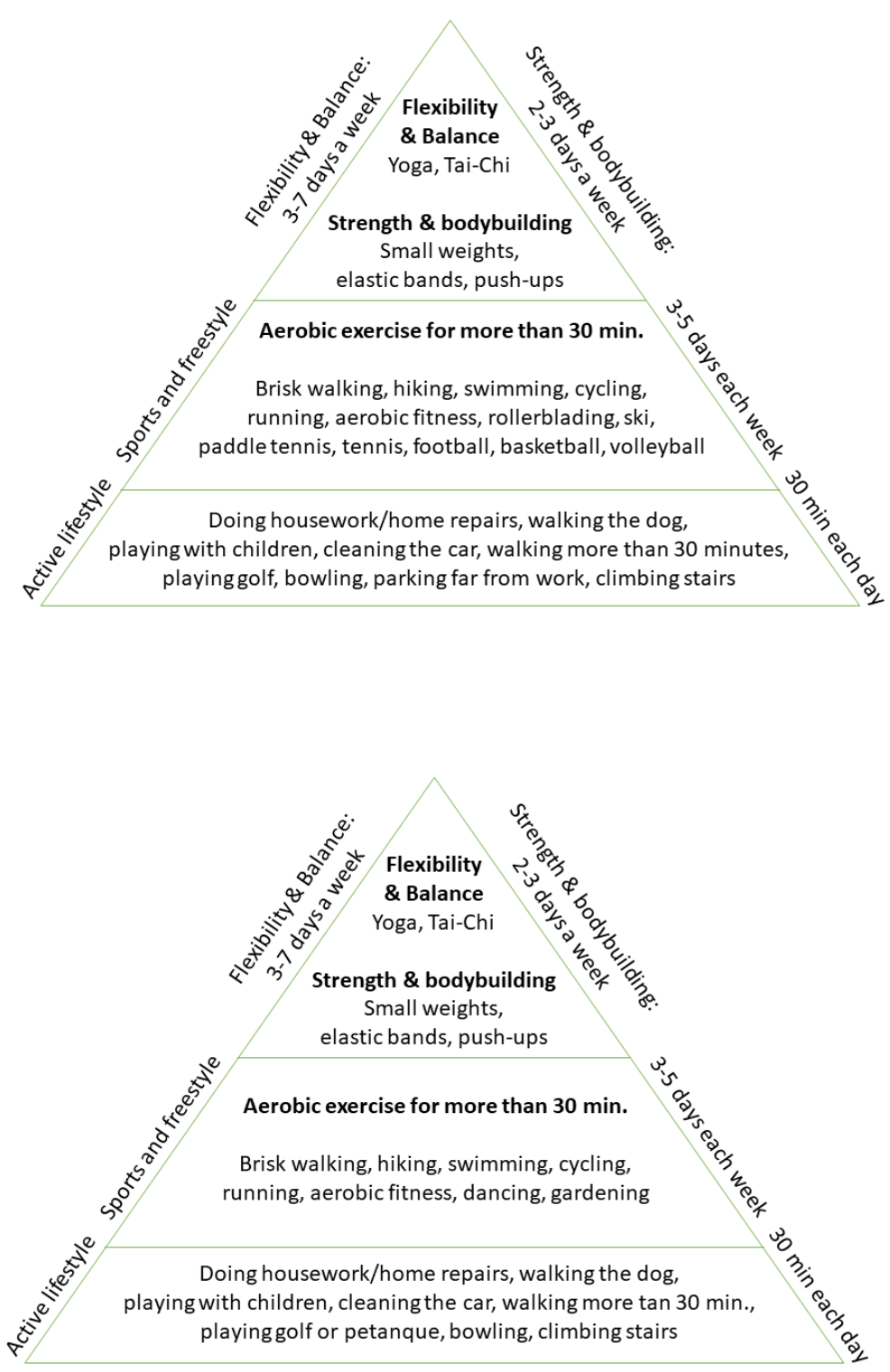


\section{References}

[1] World Health Statistics. Monograph on the Internet. Geneva: World Health Organization. Available from: https://apps.who.int/infobase/. Accessed July 27 2020.

[2] Li Y, Pan A, Wang DD, et al. Impact of Healthy Lifestyle Factors on Life Expectancies in the US Population. Circulation. 2018;138(4):345-355.

[3] Rosenstock IM. Historical Origins of the Health Belief Model. Health Educ Monographs. 1974;2:328-35.

[4] Janz NK, Becker MH. The Health Belief Model: a decade later. Health Educ Q. 1984;11(1):1-47.

[5] Ramirez FD, Chen Y, Di Santo P, Simard T, Motazedian P, Hibbert B. Association Between Self-Reported Potentially Modifiable Cardiac Risk Factors and Perceived Need to Improve Physical Health: A Population-Based Study. J Am Heart Assoc. 2017;6(5):e005491.

[6] An S, Song R. Effects of health coaching on behavioral modification among adults with cardiovascular risk factors: Systematic review and meta-analysis. Patient Educ Couns. 2020;103(10):2029-2038

[7] Chen YF, Madan J, Welton N, et al. Effectiveness and cost-effectiveness of computer and other electronic aids for smoking cessation: a systematic review and network meta-analysis. Health Technol Assess. 2012;16(38):1-205.

[8] Neville LM, O'Hara B, Milat A. Computer-tailored physical activity behavior change interventions targeting adults: a systematic review. Int J Behav Nutr Phys Act. 2009;6:30. 
[9] Neville LM, O'Hara B, Milat AJ. Computer-tailored dietary behaviour change interventions: a systematic review. Health Educ Res. 2009;24(4):699-720.

[10] Barroso M, Pérez-Fernández S, Vila MM, et al. Validity of a method for the selfscreening of cardiovascular risk. Clin Epidemiol. 2018;10:549-560.

[11] Barroso M, Zomeño MD, Díaz JL, et al. Control of cardiovascular risk factors with tailored recommendations: A randomized controlled trial. Prev Med. 2020 (in press)

[12] Schröder H, Fitó M, Estruch R, et al. A short screener is valid for assessing Mediterranean diet adherence among older Spanish men and women. J Nutr. 2011;141(6):1140-5

[13] Molina L, Sarmiento M, Peñafiel J, et al. Validation of the Regicor Short Physical Activity Questionnaire for the Adult Population. PLoS One. 2017;12(1):e0168148.

[14] Serra-Majem L, Tomaino L, Dernini S, et al. Updating the Mediterranean Diet Pyramid towards Sustainability: Focus on Environmental Concerns. Int J Environ Res Public Health. 2020;17(23):8758.

[15] Bach-Faig A, Berry EM, Lairon D, et al. Mediterranean diet pyramid today. Science and cultural updates. Public Health Nutr. 2011;14(12A):2274-84.

[16] World Health Organization. Regional Office for Physical activity factsheets for the 28 European Union member states of the WHO European region. Available at: https://www.euro.who.int/_data/assets/pdf_file/0005/382334/28fs-physicalactivity-euro-rep-eng.pdf 
[17] Marrugat J, Subirana I, Comín E, et al. Validity of an adaptation of the Framingham cardiovascular risk function: the VERIFICA Study. J Epidemiol Community Health. 2007;61(1):40-7.

[18] Martínez-González MA, García-Arellano A, Toledo E, Salas-Salvadó J, BuilCosiales P, Corella D, et al. A 14-item Mediterranean diet assessment tool and obesity indexes among high-risk subjects: the PREDIMED trial. PLoS One. 2012;7(8):e43134.

[19] Body Mass Index - BMI. Web page. Copenhagen: World Health Organization Regional Office for Europe. Available from: http:/www.euro.who.int/en/healthtopics/disease-prevention/nutrition/a-healthy-lifestyle/body-mass-index-bmi. Accessed July 272020.

[20] Shah PB. Intention-to-treat and per-protocol analysis. CMAJ. 2011;183(6):696.

[21] Estruch R, Ros E, Salas-Salvadó J, et al. Primary Prevention of Cardiovascular Disease with a Mediterranean Diet Supplemented with Extra-Virgin Olive Oil or Nuts. N Engl J Med. 2018;378(25):e34.

[22] Willett W, Rockström J, Loken B, et al. Food in the Anthropocene: the EATLancet Commission on healthy diets from sustainable food systems. Lancet. 2019;393(10170):447-492.

[23] Taylor GMJ, Dalili MN, Semwal M, Civljak M, Sheikh A, Car J. Internet-based interventions for smoking cessation. Cochrane Database Syst Rev. 2017;9(9):CD007078.

[24] Durmaz S, Ergin I, Durusoy R, Hassoy H, Caliskan A, Okyay P. WhatsApp embedded in routine service delivery for smoking cessation: effects on abstinence rates in a randomized controlled study. BMC Public Health. 2019;19(1):387. 
[25] Klein P, Lawn S, Tsourtos G, van Agteren J. Tailoring of a Smartphone Smoking Cessation App (Kick.it) for Serious Mental Illness Populations: Qualitative Study. JMIR Hum Factors. 2019;6(3):e14023.

[26] Rajani NB, Weth D, Mastellos N, Filippidis FT. Use of gamification strategies and tactics in mobile applications for smoking cessation: a review of the UK mobile app market. BMJ Open. 2019;9(6):e027883.

[27] Riegel B, Moser DK, Buck HG, et al. Self-Care for the Prevention and Management of Cardiovascular Disease and Stroke: A Scientific Statement for Healthcare Professionals From the American Heart Association. J Am Heart Assoc. 2017;6(9):e006997.

[28] Damschroder LJ, Aron DC, Keith RE, Kirsh SR, Alexander JA, Lowery JC. Fostering implementation of health services research findings into practice: a consolidated framework for advancing implementation science. Implement Sci. 2009;4:50.

[29] Walsh DMJ, Moran K, Cornelissen V, et al. The development and codesign of the PATHway intervention: a theory-driven eHealth platform for the selfmanagement of cardiovascular disease. Transl Behav Med. 2019;9(1):76-98.

[30] Conway N, Webster C, Smith B, Wake D. eHealth and the use of individually tailored information: A systematic review. Health Informatics J. 2017;23(3):218233. 


\section{FIGURE LEGENDS}

Figure 1. Flow-chart of the study

Figure 2. Prevalence of healthy lifestyles at baseline and at 1-year follow-up. Student $t$, Wilcoxon, Fisher exact and McNemar tests have been applied as appropriate.

BMI, Body Mass Index. EEPA, Energy expenditure in physical activity. MEDAS, Mediterranean Diet Adherence Score 
Table 1. Baseline characteristics, by randomization group.

\begin{tabular}{|c|c|c|c|}
\hline & $\begin{array}{l}\text { Intervention, } \\
\mathrm{N}=\mathbf{4 7 5}\end{array}$ & $\begin{array}{l}\text { Comparison, } \\
\mathrm{N}=478\end{array}$ & $P$ value \\
\hline Age (years), mean (SD) & $51(11)$ & $50(10)$ & 0.121 \\
\hline Sex (ref. women), n (\%) & $252(53.1)$ & $246(51.5)$ & 0.670 \\
\hline \multicolumn{4}{|l|}{ Education level, n (\%) } \\
\hline No studies & $7(1.5)$ & $2(0.4)$ & 0.333 \\
\hline Primary school & $102(21.6)$ & $105(22.1)$ & \\
\hline High school & $191(40.5)$ & $205(43.1)$ & \\
\hline University & $172(36.4)$ & $164(34.5)$ & \\
\hline Smoking status, $\mathrm{n}(\%)$ & & & 0.668 \\
\hline Never smoked & $210(44.3)$ & $217(45.6)$ & \\
\hline Former smoker & $160(33.8)$ & $148(31.1)$ & \\
\hline Current smoker & $104(21.9)$ & $111(23.3)$ & \\
\hline Body mass index $\left(\mathrm{kg} / \mathrm{m}^{2}\right)$, mean (SD) & $26.8(4.5)$ & $26.2(4.5)$ & 0.054 \\
\hline Waist ratio, mean (SD) & $91(13)$ & $90(13)$ & 0.378 \\
\hline Normal weight, $\mathrm{n}(\%)$ & $191(40.2)$ & $210(43.9)$ & 0.272 \\
\hline Hypertension, n (\%) & $132(28.0)$ & $125(26.4)$ & 0.647 \\
\hline Hypercholesterolemia, n (\%) & $371(78.6)$ & $361(75.8)$ & 0.349 \\
\hline Diabetes, $\mathrm{n}(\%)$ & $33(7.1)$ & $37(7.9)$ & 0.716 \\
\hline EEPA(Kcal/day), median [IQR] & 2021 [1049-3339] & $1867[1026-3315]$ & 0.444 \\
\hline Physical activity, n (\%) & & & 0.469 \\
\hline Sedentary & $155(32.6)$ & $147(30.8)$ & \\
\hline Moderate & $125(26.3)$ & $116(24.3)$ & \\
\hline Vigorous & $195(41.1)$ & $215(45.0)$ & \\
\hline MEDAS Score, mean (SD) & $7.1(2.0)$ & $6.9(1.9)$ & 0.190 \\
\hline Adherence to Mediterranean Diet, $\mathrm{n}(\%)$ & $106(22.3)$ & $96(20.1)$ & 0.445 \\
\hline
\end{tabular}

EEPA, Energy expenditure in physical activity measured with the REGICOR short questionnaire. ${ }^{10}$ IQR, Interquartile range. MEDAS, Mediterranean Diet Adherence Score measured with 14-items questionnaire. ${ }^{9} \mathrm{SD}$, Standard deviation. 
Table 2. Changes in the lifestyle with no history of cardiovascular disease at 1-year follow-up, by sex.

\begin{tabular}{|c|c|c|c|c|c|c|c|c|}
\hline \multirow{3}{*}{ Women } & \multicolumn{6}{|c|}{ Within groups analysis } & \multicolumn{2}{|c|}{ Between groups analysis } \\
\hline & \multicolumn{3}{|c|}{ Intervention, $(\mathrm{N}=\mathbf{2 5 2})$} & \multicolumn{5}{|c|}{ Comparison, $(\mathrm{N}=246)$} \\
\hline & Baseline & 12 months & $P$ value & Baseline & 12 months & $P$ value & Odds Ratio $(95 \% \mathrm{CI})$ & $P$ value \\
\hline Adherence to Mediterranean diet, $\mathrm{n}(\%)$ & $51(20.2)$ & $65(25.8)$ & 0.066 & $54(22.0)$ & $57(23.2)$ & 0.755 & $1.53(0.61-3.88)$ & 0.392 \\
\hline Normal weight, $\mathrm{n}(\%)$ & $120(47.6)$ & $117(46.4)$ & 0.505 & $132(53.7)$ & $128(52.0)$ & 0.423 & $0.90(0.10-7.03)$ & 0.999 \\
\hline Nonsmoking, $\mathrm{n}(\%)$ & $207(82.5)$ & $217(86.5)$ & 0.004 & $197(80.4)$ & $202(82.4)$ & 0.074 & -- & 0.999 \\
\hline Nonsedentary way of life, $\mathrm{n}(\%)$ & $151(59.9)$ & $148(58.7)$ & 0.779 & $156(63.4)$ & $142(57.7)$ & 0.066 & $1.57(0.66-3.79)$ & 0.315 \\
\hline \multirow[t]{2}{*}{ Men } & \multicolumn{3}{|c|}{ Intervention, $(\mathrm{N}=\mathbf{2 3 2})$} & \multicolumn{5}{|c|}{ Comparison, $(\mathrm{N}=223)$} \\
\hline & Baseline & 12 months & $P$ value & Baseline & $\mathrm{p}$-value & $P$ value & Odds Ratio $(95 \% \mathrm{CI})$ & $P$ value \\
\hline Adherence to Mediterranean diet, $\mathrm{n}(\%)$ & $55(24.7)$ & $61(27.4)$ & 0.440 & $42(18.1)$ & $46(19.8)$ & 0.651 & $1.11(0.44-2.84)$ & 0.831 \\
\hline Normal weight, $\mathrm{n}(\%)$ & $71(31.8)$ & $72(32.3)$ & 0.999 & $78(33.6)$ & $81(34.9)$ & 0.606 & $0.89(0.10-8.43)$ & 0.999 \\
\hline Nonsmoking, $\mathrm{n}(\%)$ & $163(73.1)$ & $174(78.0)$ & 0.003 & $168(72.7)$ & $174(75.0)$ & 0.149 & -- & 0.217 \\
\hline Nonsedentary way of life, $\mathrm{n}(\%)$ & $169(75.8)$ & $157(70.4)$ & 0.127 & $175(75.4)$ & $166(71.6)$ & 0.253 & $0.91(0.38-2.17)$ & 0.841 \\
\hline
\end{tabular}

Differences have been assessed with McNemar test (within group analysis) and Fisher's exact test (between group analysis)

CI, Confidence interval 
Table 3. Changes in the lifestyle at 1 -year follow-up, by age ( $<50$ and $\geq 50$ years old).

\begin{tabular}{|c|c|c|c|c|c|c|c|c|}
\hline \multirow{3}{*}{$<50$ years } & \multicolumn{6}{|c|}{ Within groups analysis } & \multicolumn{2}{|c|}{ Between groups analysis } \\
\hline & \multicolumn{3}{|c|}{ Intervention, $(\mathrm{N}=\mathbf{2 4 9})$} & \multicolumn{5}{|c|}{ Comparison, $(\mathrm{N}=261)$} \\
\hline & Baseline & 12 months & $P$ value & Baseline & 12 months & $P$ value & Odds Ratio (95\% CI) & $P$ value \\
\hline Adherence to Mediterranean diet, $\mathrm{n}(\%)$ & $44(17.7)$ & $56(22.5)$ & 0.074 & $45(17.2)$ & $47(18.0)$ & 0.874 & $1.73(0.64-4.81)$ & 0.258 \\
\hline Normal weight, n (\%) & $123(49.4)$ & $120(48.2)$ & 0.505 & $126(48.3)$ & $125(47.9)$ & 0.999 & $0.58(0.07-3.88)$ & 0.683 \\
\hline Nonsmoking, $\mathrm{n}(\%)$ & $181(72.7)$ & $194(77.9)$ & $<0.001$ & $196(75.4)$ & $202(77.4)$ & 0.114 & -- & 0.178 \\
\hline Nonsedentary way of life, n (\%) & $163(65.5)$ & $164(65.9)$ & 0.999 & $189(72.4)$ & $183(70.1)$ & 0.471 & $1.33(0.57-3.10)$ & 0.557 \\
\hline \multirow[t]{2}{*}{$\geq 50$ years } & \multicolumn{3}{|c|}{ Intervention, $(\mathrm{N}=\mathbf{2 2 6})$} & \multicolumn{5}{|c|}{ Comparison, $(\mathrm{N}=217)$} \\
\hline & Baseline & 12 months & $P$ value & Baseline & 12 months & $P$ value & Odds Ratio $(95 \% \mathrm{CI})$ & $P$ value \\
\hline Adherence to Mediterranean diet, $\mathrm{n}(\%)$ & $62(27.4)$ & $70(31.0)$ & 0.341 & $51(23.5)$ & $56(25.8)$ & 0.551 & $1.08(0.45-2.58)$ & 0.999 \\
\hline Normal weight, n (\%) & $68(30.1)$ & $69(30.5)$ & 0.999 & $84(38.7)$ & $84(38.7)$ & 0.999 & $1.31(0.14-13.3)$ & 0.999 \\
\hline Nonsmoking, $\mathrm{n}(\%)$ & $189(84.0)$ & $197(87.6)$ & 0.013 & $169(78.2)$ & $174(80.6)$ & 0.131 & -- & 0.467 \\
\hline Nonsedentary way of life, $\mathrm{n}(\%)$ & $157(69.5)$ & $141(62.4)$ & 0.027 & $142(65.4)$ & $125(57.6)$ & 0.025 & $0.97(0.38-2.46)$ & 0.999 \\
\hline
\end{tabular}

Differences have been assessed with McNemar test (within group analysis) and Fisher's exact test (between group analysis)

CI, Confidence interval 
Table 4. Changes in the lifestyle after 1-year follow-up, by education level (no studies or primary school, high school and university).

\begin{tabular}{|c|c|c|c|c|c|c|c|c|}
\hline \multirow{3}{*}{ No Studies or Primary School } & \multicolumn{6}{|c|}{ Within groups analysis } & \multicolumn{2}{|c|}{ Between groups analysis } \\
\hline & \multicolumn{3}{|c|}{ Intervention, $(\mathrm{N}=109)$} & \multicolumn{5}{|c|}{ Comparison, $(\mathrm{N}=107)$} \\
\hline & Baseline & 12 months & $P$ value & Baseline & 12 months & $P$ value & Odds Ratio $(95 \%$ CI $)$ & $P$ value \\
\hline Adherence to Mediterranean diet, $\mathrm{n}(\%)$ & $18(16.5)$ & $28(25.7)$ & 0.055 & $14(13.1)$ & $18(16.8)$ & 0.453 & $1.58(0.32-7.88)$ & 0.725 \\
\hline Normal weight, $\mathrm{n}(\%)$ & $23(21.1)$ & $26(23.9)$ & 0.450 & $31(29.0)$ & $31(29.0)$ & 0.999 & $2.32(0.16-44.94)$ & 0.592 \\
\hline Nonsmoking, $\mathrm{n}(\%)$ & $83(76.9)$ & $88(81.5)$ & 0.074 & $74(69.2)$ & $76(71.0)$ & 0.480 & -- & 0.999 \\
\hline Nonsedentary way of life, $\mathrm{n}(\%)$ & $63(57.8)$ & $60(55.0)$ & 0.663 & $71(66.4)$ & $62(57.9)$ & 0.066 & $2.06(0.46-10.19)$ & 0.333 \\
\hline \multirow[t]{2}{*}{ High School } & \multicolumn{3}{|c|}{ Intervention, $(\mathrm{N}=191)$} & \multicolumn{5}{|c|}{ Comparison, $(\mathrm{N}=205)$} \\
\hline & Baseline & 12 months & $P$ value & Baseline & 12 months & $P$ value & Odds Ratio $(95 \%$ CI $)$ & $P$ value \\
\hline Adherence to Mediterranean diet, $\mathrm{n}(\%)$ & $40(20.9)$ & $42(22.0)$ & 0.850 & $45(22.0)$ & $42(20.5)$ & 0.755 & $1.33(0.46-3.91)$ & 0.628 \\
\hline Normal weight, $\mathrm{n}(\%)$ & $79(41.4)$ & $80(41.9)$ & 0.999 & $98(47.8)$ & $98(47.8)$ & 0.999 & -- & 0.999 \\
\hline Nonsmoking, $\mathrm{n}(\%)$ & $151(79.1)$ & $157(82.2)$ & 0.041 & $157(76.6)$ & $158(77.1)$ & 0.999 & -- & 0.333 \\
\hline Nonsedentary way of life, $\mathrm{n}(\%)$ & $140(73.3)$ & $123(64.4)$ & 0.017 & $137(66.8)$ & $130(63.4)$ & 0.349 & $0.64(0.24-1.69)$ & 0.372 \\
\hline \multirow[t]{2}{*}{ University } & \multicolumn{3}{|c|}{ Intervention, $(\mathrm{N}=172)$} & \multicolumn{5}{|c|}{ Comparison, $(\mathrm{N}=164)$} \\
\hline & Baseline & 12 months & $\mathrm{p}$-value & Baseline & 12 months & $\mathrm{p}$-value & & p-value \\
\hline Adherence to Mediterranean diet, $\mathrm{n}(\%)$ & $48(27.9)$ & $56(32.6)$ & 0.280 & $37(22.6)$ & $43(26.2)$ & 0.345 & $0.95(0.32-2.81)$ & 0.999 \\
\hline Normal weight, $\mathrm{n}(\%)$ & $89(51.7)$ & $83(48.3)$ & 0.077 & $81(49.4)$ & $80(48.8)$ & 0.999 & $0.20(0.01-2.84)$ & 0.294 \\
\hline Nonsmoking, $\mathrm{n}(\%)$ & $133(77.3)$ & $143(83.1)$ & 0.004 & $133(82.1)$ & $141(86.5)$ & 0.043 & -- & 0.481 \\
\hline Nonsedentary way of life, $\mathrm{n}(\%)$ & $115(66.9)$ & $121(70.3)$ & 0.405 & $121(73.8)$ & $115(70.1)$ & 0.417 & $1.91(0.69-5.37)$ & 0.245 \\
\hline
\end{tabular}

Differences have been assessed with McNemar test (within group analysis) and Fisher's exact test (between group analysis)

CI, Confidence interval 


\section{Figure 1.}

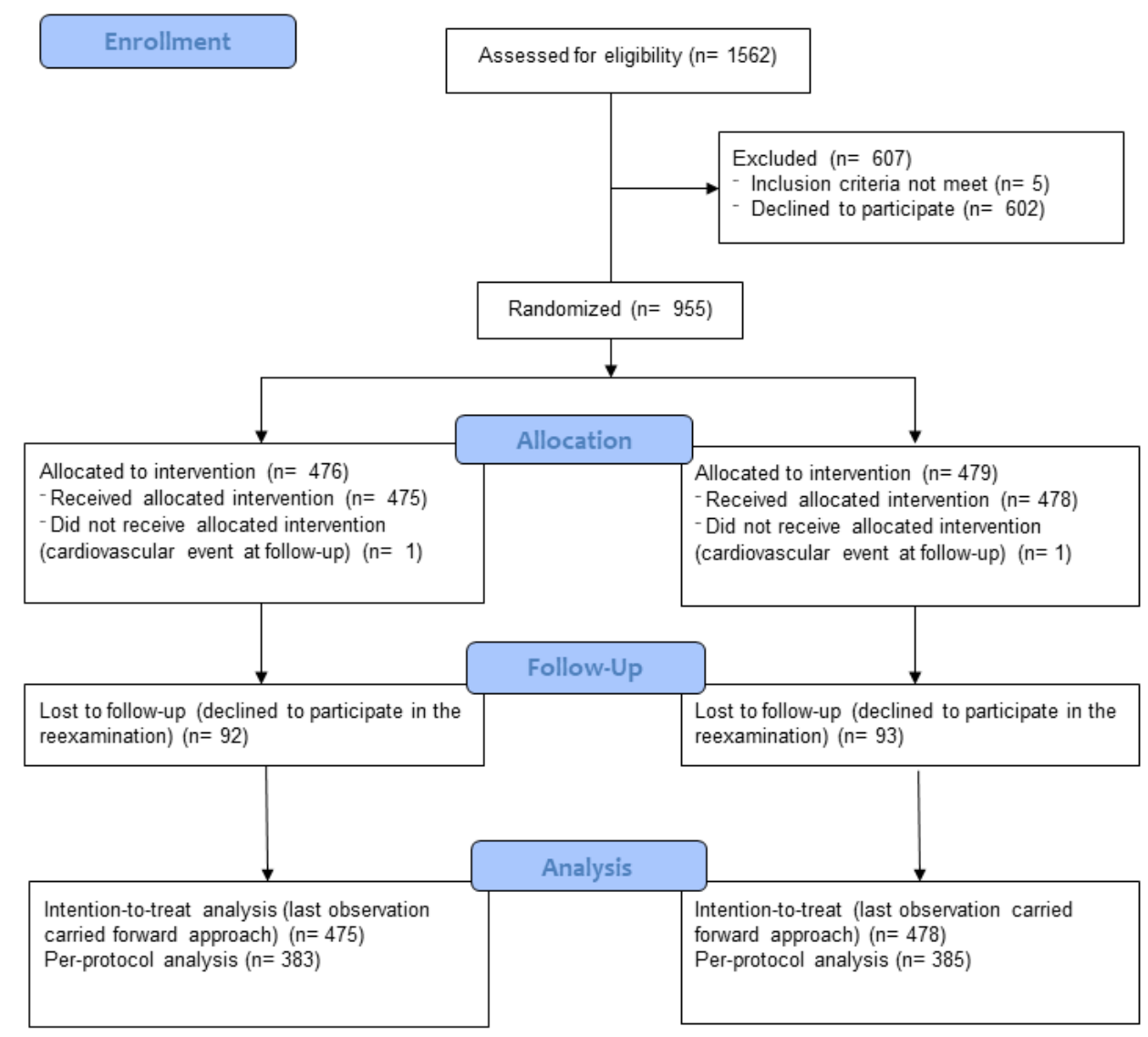


Figure 2.
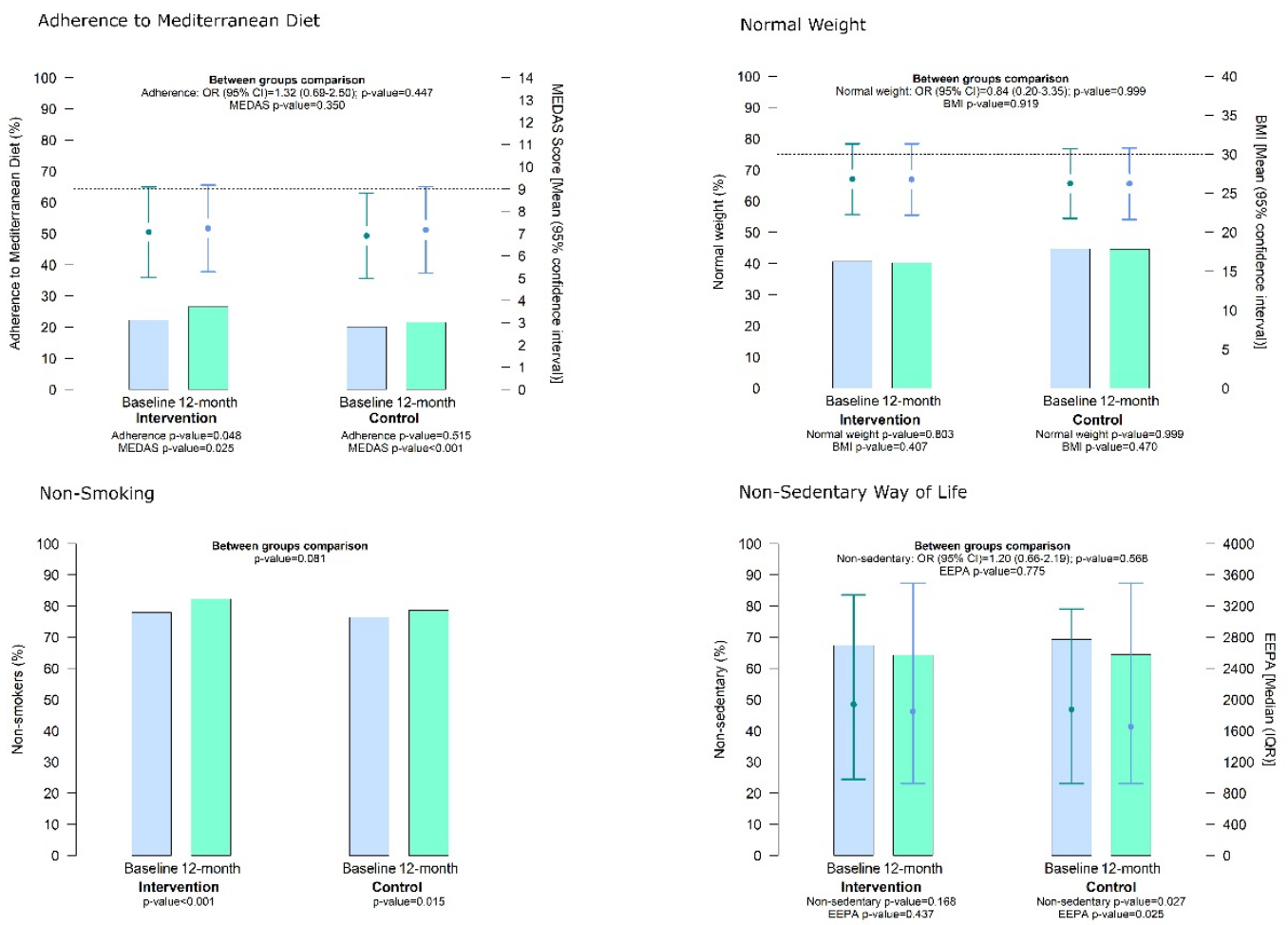